\title{
Pratiques
}

Linguistique, littérature, didactique

147-148 | 2010

Interactions et Corpus Oraux

\section{Alain Rabatel, Les reformulations pluri-sémiotiques en contexte de formation}

Presses universitaires de Franche-Comté, 2010

\section{Anne Leclaire-Halté}

\section{(2) OpenEdition}

\section{Journals}

Édition électronique

URL : http://journals.openedition.org/pratiques/1675

DOI : 10.4000/pratiques. 1675

ISSN : 2425-2042

Éditeur

Centre de recherche sur les médiations (CREM)

Édition imprimée

Date de publication : 15 décembre 2010

Pagination : 244-248

\section{Référence électronique}

Anne Leclaire-Halté, " Alain Rabatel, Les reformulations pluri-sémiotiques en contexte de formation »,

Pratiques [En ligne], 147-148 | 2010, mis en ligne le 15 décembre 2010, consulté le 22 septembre 2020. URL : http://journals.openedition.org/pratiques/1675; DOI : https://doi.org/10.4000/pratiques.1675 


\section{Note de lecture}

\section{Les reformulations pluri-sémiotiques en contexte de formation}

Ouvrage collectif sous la direction d'Alain Rabatel

Presses universitaires de Franche-Comté, 2010

Comme le rappelle Alain Rabatel dans l'introduction de cet ouvrage de 298 pages (dont 15 de bibliographie), des évolutions significatives touchent aujourd'hui les situations de formation initiale ou continuée, notamment avec le recours aux technologies modernes. Ces évolutions invitent à prendre en compte, dans ces situations de formation, la plurisémioticité qui les caractérise. La spécificité de cet ensemble de treize chapitres (en comptant 1'introduction d'A. Rabatel) qui est proposé à notre lecture réside dans l'analyse des reformulations liées aux activités scolaires, non limitées au niveau verbal et incluant images, schémas, dessins, gestes liés aux activités, prenant en compte aussi le caractère multimodal des discours oraux accompagnant les dispositifs sémiotiques utilisés.

Ce volume est donc original à deux titres :

- il propose une approche élargie de la reformulation en incluant ses rapports avec multi-modalité et pluri-sémioticité (la reformulation, dans l'introduction, est définie comme «métaterme englobant l'ensemble des activités langagières par lesquelles le locuteur/énonciateur revient sur des dires antérieurs $\gg)$;

- il présente uniquement des analyses de corpus donnés comme didactiques, avec comme objectif de faire évoluer la formation par l'approche 
linguistique ; la variété des situations de formation évoquées (de la lecture d'un ouvrage au cours magistral à l'université en passant par le cours au collège, à l'école primaire, en FLM, FLE), ainsi que celle des apprenants (adultes, étudiants, élèves du secondaire ou du primaire), est à souligner.

Nous avons fait le choix d'un compte rendu linéaire devant la diversité des chapitres, qu'Alain Rabatel, dans son introduction, regroupe en deux parties.

La première illustre la complexité et la polyvalence des reformulations selon les sémioses. La première contribution, celle d'A. Rabatel, « Dire et montrer : quand les schémas reformulent le texte », est la seule à ne pas s'appuyer sur un corpus oral et inclut dans les situations de formation la lecture d'ouvrages théoriques. Elle étudie, dans quatre ouvrages de J.-M. Adam, susceptibles d'être lus tant par les étudiants que par les enseignants, la reformulation dans le rapport entre texte et schéma. La conclusion est qu'avec le schéma, pivot entre texte reformulé et texte à commenter/reformuler, la reformulation n'apparaît plus seulement comme un outil de clarification, mais elle est aussi opacifiante : elle interroge les représentations et les questionnements des concepts, en ne relevant pas simplement d'explicitations mais en impliquant des changements de point de vue.

Ensuite, avec « Postures énonciatives et marqueurs de reformulation en situation de conférence avec diaporama. Alors, effectivement, donc » de Claire Doquet-Lacoste et Elisabeth Richard, le lecteur passe à d'autres dispositifs sémiotiques dans une autre situation de formation : celle du colloque. Leur corpus, réunissant un article scientifique, un diaporama sur power point et la communication orale accompagnant la présentation du diaporama, leur permet d'étudier les reformulations d'un discours à l'autre, ainsi que les reformulations de discours autres au sein de ces trois discours. Les connecteurs alors, effectivement, donc, sont caractérisés dans leur relation à la situation pluri sémiotique associant diaporama et communication orale et l'accent est mis sur le statut énonciatif particulier des énoncés sur diaporama, présents à l'écrit en même temps que reformulés oralement.

La contribution suivante, due à Jean-Charles Pochard, «Les reformulations et altérations d'une citation, à travers ses avatars pluri sémiotiques », part également d'une situation de formation impliquant le recours au power point : une conférence à des étudiants de didactique des langues. J.-C. Pochard propose l'analyse d'une citation tirée du Cadre Européen Commun de Référence pour les langues puis montre comment cette citation est traitée, par le même auteur, successivement dans un article de revue (Les Langues modernes), dans le power point élaboré à partir de l'article en articulation avec un exposé oral, puis enfin dans cet exposé oral. Son analyse montre comment la plurisémioticité des situations de communication, avec les reformulations et les altérations successives qu'elle entraine, déplace des notions tout en durcissant progressivement la position de l'auteur par rapport à la citation originelle.

Robert Bouchard et Chantal Parpette, quant à eux, dans le cadre de leurs travaux sur le cours magistral universitaire ( Reformulations, coformulation et dispositifs de coformulation : l'exemple du cours magistral scientifique »), mettent plus l'accent que les auteurs des deux articles précédents sur la ques- 
tion de la réception des discours de formation par leur destinataire, les étudiants. Ils s'intéressent particulièrement à la notion de coformulation comme " effet sur un public à qui on expose simultanément deux productions signifiantes constituant un seul message ». Ils étudient deux types de dispositifs de coformulation lors d'un cours magistral de thermodynamique. Après avoir souligné que, dans le cours magistral, le passage qu'effectue l'enseignant de ses notes à l'oral rend difficile, pour les étudiants, l'appropriation cognitive, ils reviennent sur les moyens auxquels l'enseignant peut recourir pour aider à cette appropriation et étudient deux types de coformulation. Le premier est lié à la coprésence d'un document iconique fixe et d'un discours oral. Dans ce cas, contrairement à la situation de lecture d'ouvrage qu'évoque A. Rabatel, le schéma est le texte source et le discours de l'enseignant l'énoncé reformulant. Les reformulations orales permettent d'éclairer les informations elliptiques et pragmatiquement non situées du document projeté, mais l'étudiant a la liberté de lire le document projeté comme il l'entend. Le second dispositif de coformulation est l'association de la projection d'une page d'écran, et, de façon simultanée, la construction d'un graphe et la production verbale qui l'accompagne : dans cette association de deux processus sémiotiques synchrones, on ne peut distinguer ni reformulé ni reformulant.

L'article suivant écrit par Simona Pekarek Doehler et Evelyne PochonBerger s'intitule « La reformulation comme technique de gestion du désaccord : le format tying dans les interactions en classe de langue ». Situé dans le cadre de l'analyse conversationnelle, il se place encore plus du côté des apprenants et présente un type spécifique de reformulation, le format tying, ressource pour gérer le désaccord. Le format tying reprend le format d'un énoncé antérieur en jouant sur les formatages lexico-syntaxiques, prosodiques aussi bien que gestuels et exploite ainsi des ressources multimodales. Qu'en est-il dans les interactions en classe de langue ? Les données consistent en 92 heures d'enregistrements audio/vidéo en cours de L1 et L2 avec des élèves de 13-14 ans et 17-18 ans. L'article montre les trois fonctions de ce type de reformulation, qui peut être opérateur de désaccord immédiat, préface modératrice au désaccord (par une reformulation de type oui mais), enchainement contrastif à distance.

Dans tous les cas, un sens nouveau est donné aux propos repris, par le fait même que l'auteur de l'énonciation change, et les auteures de l'article insistent sur la créativité communicative et les compétences interactives des adolescents.

Cette première partie se clôt sur la contribution de Hugues de Chanay et Denis Vigier (« Voulez-vous reformuler avec moi ? Approche interactive et polysémiotique de la reformulation en interaction didactique »). Résultant de l'étude de quatre vidéos tournées en collège, elle présente pour nous un triple intérêt :

- elle propose une typologie des réénonciations dont les reformulations font partie et définit clairement une série de concepts en rapport avec ces dernières ;

- elle donne une vision étendue de la reformulation en prenant en compte des reformulations effectuées partiellement sur un mode non verbal et en 
indiquant que leur portée peut varier (micro, méso, macro);

- elle met au service de la dimension didactique l'étude de la reformulation dans les séances filmées : elle note que «plus une reformulation relève d'un niveau élevé, plus elle est informée par les visées poursuivies par l'enseignant pendant le cours » et que la reformulation didactique vaut plus pour le parcours qu'elle permet d'effectuer que pour la formulation finale, étant donné que l'enseignant amène les élèves, dans le cas du corpus analysé, à une formulation déjà existante.

La seconde partie de l'ouvrage met l'accent sur l'articulation entre reformulation et construction des savoirs, en articulant reformulation et place institutionnelle des reformulateurs. C'est ainsi que Laetitia Peifer (« La restitution de fictions pluri sémiotiques entre pairs : des reformulations imitatives aux reformulations explicatives ») étudie, chez des élèves de CE1 et CE2, la reformulation des textes sources polysémiotiques que sont la série animée Il était une fois la vie et trois albums de Pef (mettant en scène le Prince de Motordu). Elle distingue trois unités d'analyse dans les textes cibles : la reformulation proprement dite, les entours de la reformulation et la co-reformulation entre pairs (particulièrement à partir des albums de Pef).

De la reformulation imitative à la reformulation explicative, les stratégies des élèves sont multiples et l'auteure note que ce sont les images qui sont d'abord reformulées. Quant à la co-reformulation entre pairs, elle est particulièrement présente pour les albums de Pef, dont la sémiotique appelle différentes lectures. En conclusion, 1'objet support du texte-source et le texte-source sont indissociables et les stratégies de reformulation sont en relation avec la réception qu'appelle le texte-source.

Le lecteur reste à l'école élémentaire avec Nathalie Blanc et Peter Griggs dans « Reformulations et apprentissages dans le contexte plurisémiotique d'une classe de langue étrangère ". Ils analysent la première phase d'apprentissage de l'anglais, la reconstitution orale d'une chanson et d'un mime, et montrent que les composantes non linguistiques s'inscrivent dans le schéma canonique de la reformulation et ont trois fonctions : déclencheur marqueur de la reformulation, étayage de l'énoncé doublon et enchâssement d'un travail métalinguistique. Les auteurs concluent sur la différenciation entre les composantes non linguistiques, selon que la reconstitution textuelle va du sens à la forme (mime) ou de la forme au sens (chanson).

Kristine Lund et Karine Bécu-Robinault, elles, s'intéressent à la physique dans «La reformulation multimodale et polysémiotique comme aide à la compréhension de la physique ». Elles montrent le lien entre les rôles socio-institutionnels des acteurs et les reformulations multimodales et polysémiotiques : des professeurs stagiaires reformulant les gestes et les verbalisations d'élèves observés sur vidéo et un professeur dans sa classe n'ont pas la même perception des objectifs d'apprentissage. Elles montrent également comment les semiotic bundles (Arzarello 2004) évoluent grâce aux reformulations plurisémiotiques et multimodales, et l'importance de ces dernières pour évaluer la distance sémantique entre le savoir enseigné et les connaissances des élèves.

Et la reformulation dans le cadre du cours d'EPS ? Dans «Plurisémioticité 
et pluri-sémioticité en Education Physique et Sportive », Nathalie Wallian, après avoir rappelé son cadre théorique, à la croisée des philosophies de l'action et de la théorie des situations didactiques, donne deux exemples de reformulation pluri-sémiotique d'une action : une action filmée commentée par gestes et paroles, une combinaison paroles, gestes et dessin. Elle montre les fonctions cognitives, interactionnelles et actionnelles de ces reformulations et l'importance des interactions pluri-modales et pluri-sémiotiques en didactique de l'EPS : «elles engagent des processus langagiers spécifiques et produisent des effets pragmatiques sur l'apprentissage ».

Les deux derniers articles ont trait à la formation professionnelle. Ecaterina Bulea, dans « Signes, discours et interprétation de l'agir : le rôle des reformulations dans des entretiens portant sur le travail infirmier», explique d'abord pourquoi la notion d'invariant sémantique, à propos de la reformulation, est remise en cause pour une conception plus élargie, puis développe les apports de la théorie saussurienne à l'étude de la reformulation. Elle analyse un corpus d'entretiens avec des infirmières à propos de l'effectuation d'un soin et en tire trois conclusions. Tout d'abord, la reformulation n'a pas qu'une fonction de réparation, elle sert aussi à la construction d'une pluralité d'angles de saisie de l'agir. Ensuite, il est souvent difficile de circonscrire le segment reformulé, une reformulation pouvant avoir plusieurs points d'ancrage. Enfin, E. Buléa avance une définition de la reformulation comme «processus d'instauration discursive d'un rapport de proximité entre valeurs [au sens saussurien du terme [...] portées par des entités d'empan différent ».

Enfin, Laurent Filliettaz, Ingrid de Saint Georges, Barbara Duc, après un exposé très éclairant sur la nécessité, aujourd'hui, en contexte didactique, de sortir d'une conception verbocentrique de la reformulation, pour définir les processus reformulatifs comme des opérations de resémiotisation dans des trajectoires situées d'apprentissage, proposent une étude de cas. Dans « Reformulation, sémiotisation et trajectoires d'apprentissage en formation professionnelle initiale : l'enseignement du giclage du mortier en maçonnerie », ils montrent, dans ce domaine spécifique de l'enseignement professionnel, l'usage et les effets des processus reformulatifs, tant chez l'enseignant que chez les apprenants.

En conclusion, cet ouvrage, qui enrichit, actualise nos savoirs sur la reformulation, montre l'intérêt d'une conception élargie des processus reformulatifs en contexte didactique. Il invite à poursuivre la réflexion tant sur le rôle de la reformulation dans les apprentissages des apprenants que sur les compétences qu'elle requiert chez les enseignants : comment mettre tous ces savoirs au service de la formation des enseignants?

Anne LECLAIRE-HALTÉ 